\title{
Antibiotics I: new antibiotics and advances in antibiotic treatment
}

\author{
C R PENNINGTON，J CROOKS
}

The prescribing of antibiotics has been influenced by the increasing number of available drugs, changing patterns of bacterial resistance and disease, a changing patient population, and the patient's belief that all infections respond to antibiotics. In this article we review developments in antibiotic prescribing which have followed the introduction of new drugs and the recognition of "new" applications for established ones.

\section{New antibiotics}

New antibiotics may conveniently be considered in four main groups: penicillins, cephalosporins, aminoglycosides, and a miscellaneous group.

\section{PENICILLINS}

The introduction of new penicillin analogues and esters, new derivatives of carbenicillin, the acylureido penicillins (azlocillin, mezlocillin, and piperacillin), and mecillinam has provided a range of drugs that share the features of relative safety, occasional hypersensitivity, and susceptibility to inactivation by bacterial $\beta$-lactamase with the parent compound. One innovation of interest is the combination of the $\beta$-lactamase inhibitor clavulanic acid with one member of the group amoxycillin (Augmentin) in order to extend its antibacterial spectrum, and developments of this type might conceivably be applied to other members of the group.

\section{The ampicillins}

The insertion of a hydroxyl group in the benzyl side chain of ampicillin resulted in the production of amoxycillin. Compared with ampicillin amoxycillin (Amoxil) has several advantagesnamely, improved intestinal absorption with correspondingly greater tissue concentrations and a considerably more rapid cidal action. Diarrhoea and the selection of resistant organisms occur less often during treatment with amoxycillin. These advantages together with the fewer daily doses required than

Ninewells Hospital and Medical School, Dundee DD1 9SY

C R PENNINGTON, MD, MRCP, consultant physician and honorary senior lecturer in medicine

J CROOKS, MD, FRCP, professor of therapeutics with ampicillin have led to this derivative being increasingly used.

Ampicillin esters such as talampicillin (Talpen) have no antibacterial activity, but ampicillin is liberated by hydrolysis during absorption. The lower incidence of diarrhoea and reduced selection of resistant organisms must be weighed against the tendency of these preparations to provoke gastric disturbance and the knowledge that ampicillin rather than an improved analogue is the actual antibiotic. Consequently these derivatives would appear to confer no advantage.

The combination of clavulanic acid with amoxycillin (Augmentin) extends the activity of amoxycillin against organisms which owe their resistance to the production of $\beta$-lactamase enzymes. These include Staphyloccus aureus, many species of Enterobacteriaceae, Neisseria gonorrhoeae and resistant forms of Haemophilus influenzae. Experience with Augmentin is presently limited to infections of the urinary and respiratory tracts. Adverse reactions occur in $12 \%$ of recipients and mainly consist of nausea, vomiting, and diarrhoea. At present it is recommended that this drug is reserved for use against respiratory and urinary infections caused by organisms known to be resistant to amoxycillin alone.

\section{Carfecillin and ticarcillin}

Carbenicillin has a predominantly Gram negative spectrum of activity that includes Pseudomonas aeruginosa and some other bacilli resistant to ampicillin. Large doses are required by intravenous infusion, which creates the potential problem of sodium overload. Carfecillin (Uticillin) is the phenyl ester of carbenicillin which is absorbed after oral administration and hydrolysed by tissue esterases to phenol and carbenicillin. Unfortunately, tissue concentrations of the active drug are low although urinary concentrations are adequate. Carfecillin is, therefore, reserved for use against urinary tract infections, especially those due to Ps aeruginosa.

Ticarcillin (Ticar) resembles carbenicillin in its pharmacology and range of antimicrobial activity except that it is twice as active against $P$ s aeruginosa. It is not, however, active in vivo against strains resistant to carbenicillin. Nevertheless, ticarcillin's greater activity represents an important advantage over carbenicillin.

\section{Azlocillin, mezlocillin, and piperacillin}

Azlocillin (Securopen) is a new parenteral acylureido penicillin. Against Ps aeruginosa it is two to four times more active than ticarcillin or mezlocillin, and it can be active against strains resistant to other agents. Like carbenicillin and ticarcillin it is less active than mezlocillin against Escherichia coli and Klebsiella spp. At present it would seem reasonable to 
reserve azlocillin for use against a strain of $P s$ aeruginosa known to be resistant to other agents.

Mezlocillin (Baypen) embraces the spectrum of ampicillin and carbenicillin and has a broader spectrum than azlocillin. Although it has been advocated for treating a wide range of infections, in vitro studies suggest that bacterial resistance, especially with coliforms and anaerobes, may be a problem. Consequently, it should not be used alone for abdominal sepsis; for this condition combination with an aminoglycoside such as gentamicin or netilmicin is recommended.

Piperacillin (Pipril) is the most recent addition to this group. It is claimed that it combines the potency of azlocillin against Ps aeruginosa with the broad spectrum of mezlocillin. It is active against some strains of Ps aeruginosa resistant to other agents and a wide range of micro-organisms, including some strains of bacteroides. Piperacillin is therapeutically active against organisms producing $\beta$-lactamases, to which it is unstable in vitro, and clinical studies have shown that it may be effective in serious infections when used alone. It should, however, be used in conjunction with another antibiotic, such as an aminoglycoside, as resistance may develop.

Further studies are needed to define more precisely the role of these drugs. At present they are known to be useful antipseudomonal agents and they exhibit synergy with aminoglycosides, with which they should be combined to treat serious intra-abdominal infection.

\section{Mecillinam}

Mecillinam is a novel derivative with a different mode of action on the cell wall and a Gram negative spectrum. Mecillinam (Selexidin) is not absorbed; the oral form is the ester pivmecillinam (Selexid). The main role of this drug is in managing urinary infections, for which it is safe and effective and active against many organisms resistant to amoxycillin. Although it exhibits impressive in vitro activity against Salmonella species, results in patients with enteric fever have been disappointing, and the drug has no established place in the management of systemic infections.

\section{CEPHALOSPORINS}

The cephalosporins are traditionally classified into three "generations." The first generation includes drugs such as cephalexin, cephradine, cephaloridine, and cefaclor. All drugs in this group lack stability to $\beta$-lactamase enzymes elaborated by Gram negative organisms, and they have no action against Ps aeruginosa. They have been available for many years and will be considered no further. The second generation includes cefuroxime, cefoxitin and cephamandole. These drugs differ from the first generation group by virtue of their stability to $\beta$ lactamases. Stability to $\beta$-lactamases is also a feature of the third generation. Members of this group are distinguished by their activity against $P$ s aeruginosa. So far cefotaxime, cefsulodin, and latamoxef disodium have been marketed.

\section{Second generation cephalosporins}

Cefuroxime (Zinacef) has useful activity against $S$ aureus, $H$ influenzae, and many Gram negative bacteria, including penicillin resistant $N$ gonorrhoeae. Compared with cefuroxime cefoxitin (Mefoxin) has reduced activity against some Gram positive organisms but a spectrum of activity that includes anaerobes. Cephamandole (Kefadol) is not so resistant to $\beta$ lactamase activity compared with cefuroxime and cefoxitin Consequently, cefuroxime is the best broad spectrum drug in this group except for infections caused by anaerobes, when cefoxitin may be preferred.

\section{Third generation cephalosporins}

Third generation cephalosporins are characterised by a spectrum of activity that includes Ps aeruginosa, long half lives, and poor penetration of the central nervous system. Cefotaxime (Claforan) has slightly lower activity against $P$ s aeruginosa but activity against some anaerobes. Unlike most cephalosporins cefotaxime is metabolised in vivo, so reduced dosage is required only in severe renal failure. Cefsulodin (Monaspor) is a relatively narrow spectrum antipseudomonal drug, and this property is a potential advantage in treating infections caused by this organism. Latamoxef disodium (Moxalactam) has a spectrum that includes Gram negative and anaerobic organisms. It may have a role in surgical prophylaxis. Many more drugs such as ceftazidene and cefoperazone are presently being evaluated. The role of these newer agents has not yet been clarified.

\section{AMINOGLYCOSIDES}

Aminoglycosides exhibit impressive activity against Gram negative organisms including $P s$ aeruginosa but relatively poor activity against some Gram positive species, especially streptococci. Toxicity is their main disadvantage. Nephrotoxicity may be enhanced by the concomitant use of cephalosporins (including the newer agents) but not the penicillins. Ototoxicity may be primarily vestibular (gentamicin, tobramycin, and netilmicin) or auditory (streptomycin, kanamycin, and amikacin). New developments have included the introduction of derivatives that are less toxic and less prone to destruction by bacterial enzymes.

Tobramycin (Nebcin) is considered to be more active against Ps aeruginosa and less nephrotoxic than gentamicin. Amikacin (Amikin) is resistant to all but a few of the enzymes that destroy aminoglycosides and may therefore retain activity against bacteria resistant to other agents. This property is shared with netilmicin (Netillin), which is also less toxic. Unlike gentamicin and tobramycin, amikacin and netilmicin are not inactivated by carbenicillin and ticarcillin in the presence of renal failure.

Although the use of aminoglycosides has decreased after the introduction of the second and third generation cephalosporins, they remain valuable drugs and exhibit synergy with the acylureido penicillins. This combination is particularly useful in managing unknown serious infection, particularly in the immunosuppressed patient. The increased safety of netilmicin suggests that this drug should replace older derivatives. Amikacin should be reserved for infections known to be resistant to other aminoglycosides.

\section{MISCELLANEOUS ANTIBIOTICS}

Co-trimoxazole contains fixed combinations of sulphamethoxazole and trimethoprim that sequentially inhibit bacterial folate metabolism and exhibit synergy in the ratio of $20: 1$. Whereas this ratio occurs in the blood after absorption, in the urine the ratio of the two components is $1: 1$. Under these circumstances there is no synergy, and antibacterial activity is largely due to the trimethoprim moiety. This observation has led to two developments; firstly, the use of other sulphonamides in combination with trimethoprim for the treatment of urinary tract infections-for instance, sulphadiazine as co-trimazine and sulphametrol as Lidaprim-and secondly, the marketing of trimethoprim alone. Trimethoprim alone is attractive because many bacterial pathogens are now resistant to sulphonamides, and the sulphonamide component of co-trimoxazole is responsible for most adverse reactions-for example, rashes and blood dyscrasias as well as drug interreactions such as those with warfarin and sulphonylureas. Clinical trials have shown that trimethoprim (Monotrim, Syraprim, Ipral, Trimopan) is as effective as co-trimoxazole in treating urinary tract infections and some forms of respiratory infections. New derivatives of 
trimethoprim are presently being evaluated. Concern has been expressed that monotherapy may encourage the emergence of bacterial resistance, but this contention is as yet unsubstantiated. Trimethoprim should be used in preference to co-trimoxazole in treating urinary tract infections in patients who are intolerant of sulphonamides as well as those receiving drugs with which sulphonamides may interreact.

Cinoxacin and rosoxacin are derivatives of malidixic acid. Cinoxacin is more active in vitro than nalidixic acid, and rosoxacin has useful activity against $N$ gonorrhoeae. Both are effective against most urinary pathogens. Like the parent compound they achieve inadequate blood and tissue concentrations, and their use should be restricted to treating "lower" urinary tract infections.

Finally, tinidazole (Fasigyn) has a similar spectrum of activity to metronidazole. It may, however, be given as a single daily dose.

\section{New applications}

\section{TREATMENT OF ESTABLISHED INFECTIONS}

Legionnaire's disease has attracted much interest. Retrospective clinical data have clearly indicated that the best therapeutic response was obtained in patients who were treated with erythromycin. The need for high dosage intravenous therapy has been emphasised. More recently, the impressive in vitro activity of rifampicin against the legionella bacillus has been recognised, and now the combined use of erythromycin and rifampicin is recommended for managing legionnaire's disease.

Another unusual respiratory pathogen is Pneumocystis carinii, which is found increasingly in the immunosuppressed patient. In high dosage co-trimoxazole is effective with results similar to those obtained with pentamidine.

Once the role of Clostridium difficile in the development of postantibiotic diarrhoea and pseudomembranous colitis was recognised oral vancomycin was found to be effective in these conditions. Previously, vancomycin was known to be effective but toxic after systemic administration, which was usually reserved for intractable staphylococcal infections; absorption and therefore toxicity are not associated with oral administration. Another recently recognised intestinal infection is campylobacter enteritis which, unlike other forms of bacterial gastroenteritis, responds to antibiotics. Erythromycin is the drug of choice, but its use should be reserved for those patients with bacteraemia or severe disease. Indeed, one study has shown that no benefit accrues from the routine administration of erythromycin in this condition.

An important advance in managing various forms of endogenous infection, especially postoperatively, has followed the recognition of the importance of anaerobic bacteria. Metronidazole, previously used against protozoal infections, was found to be effective against such pathogens, for which purpose it replaced clindamycin. Recent reports have indicated that metronidazole may have a role in the treatment of Crohn's disease.

Finally, the tradition of present dose schedules has been questioned. The use of single large dose pulse therapy appears to be of some value in the treatment of lower (but not upper) urinary tract infections. Whereas such use has many theoretical advantages-such as reducing drug costs, adverse reactions, and antibiotic resistance-selecting appropriate patients for single dose treatment remains a problem. This treatment, however, is reasonable in patients with frequency and dysuria in the absence of pyrexia and loin pain; treatment failure or relapse both indicate the need for a conventional course of antibiotics.

\section{ANTIBIOTIC PROPHYLAXIS}

Developments in the prophylactic use of antibiotics have occurred in three areas. Firstly, sulphonamides are no longer of value for prophylaxis against meningococcal infections in close contacts because $30^{\circ}$ of the organisms are now resistant to such drugs. Instead, rifampicin may be used in divided doses for two days. Minocycline is an alternative choice, but it has been associated with unpleasant adverse reactions. Secondly, the use of oral non-absorbed antimicrobial agents such as framycetin, colistin, and nystatin may considerably reduce endogenous infections in neutropenic patients. Similar claims have been made for co-trimoxazole.

Nevertheless, the major developments have taken place in surgical sepsis. Administration of antibiotics immediately before operation greatly reduces the incidence of surgical sepsis in patients undergoing operations for gastric outflow obstruction, biliary tract disease, and disease of the colon and uterus. Under these circumstances the combination of metronidazole with a second generation cephalosporin or aminoglycoside is favoured, and the preoperative dose is followed by up to two postoperative injections. By contrast, it is important to provide cover against Staph aureus and clostridia during some orthopaedic and vascular surgery, and these patients receive treatment for up to five days postoperatively. Benzylpenicillin is the drug of choice for prophylaxis against clostridia while flucloxacillin provides cover against Staph aureus. Erythromycin is a suitable alternative in those patients allergic to penicillin.

The wide range of available drugs and changing patterns of bacterial resistance have created problems of drug evaluation and prescribing. Whereas there are data on the potential and actual clinical efficacy of many new agents, their relative merits in different clinical circumstances have yet to be satisfactorily evaluated. Furthermore, their availability brings the hazard of inappropriate or unnecessarily expensive prescribing by clinicians, who may find difficulty in keeping abreast with current developments. This problem is being met in some areas by the introduction of antibiotic policy documents that provide locally generated information and a consensus view on the use of antibiotics to achieve cost effective prescribing.

\section{Bibliography}

Anders BJ, Lauer BA, Paisley JW, Reller LB. Double blind placebo controlled trial of erythromycin for treatment of campylobacter enteritis. Lancet $1982 ; 1: 131-2$.

The routine use of erythromycin was evaluated in unselected patients with campylobacter gastroenteritis.

Anderson JD, Adams MA, Barrington JC. Comparison of the epidemiology of bacterial resistance to mecillinam and ampicillin. Antimicrob Agents Chemother 1976;10:872-7.

Reviews patterns of drug resistance in coliforms causing urinary tract infection with particular reference to the use of ampicillin and mecillinam.

Baily RR. The aminoglycosides. Drugs $1981 ; 22: 321-7$.

Antimicrobial activity, toxicity, and clinical use of established and new aminoglycoside derivatives are discussed.

Brogden RN, Carrmine A, Heel RC, Morley PA, Speight TM, Avery GS. Amoxycillin-clavulanic acid: a review of its antibacterial activity, pharmacokinetics, and therapeutic use. Drugs $1981 ; 22: 337-62$.

A review of current experience.

Coppens L, Klastersky J. Comparative study of anti-pseudomonas activity of cyclocillin, mezlocillin and ticarcillin. Antimicrob Agents Chemother $1979 ; 15: 396-9$.

Compares the antipseudomonal activity of some new penicillin derivatives.

Cuhna BA, Ristuccia AM. Third generation cephalosporins. Med Clin North Am $1982 ; 66: 283-91$.

The antimicrobial spectrum and potential role of forthcoming third generation cephalosporins are reviewed.

Feathers RS, Lewis AA, Sager GR, Amirak ID, Noone P. Prophylactic systemic antibiotics in colorectal surgery. Lancet 1977;ii :4-8.

The value of perioperative antibiotics in reducing postoperative sepsis was studied in patients undergoing colonic surgery.

Fu KP, Ness HC. Azlocillin and mezlocillin: new ureido penicillins. Antimicrob Agents Chemother 1978;13:930-8.

The antibacterial spectrum and clinical applications of new ureido penicillins are described.

Hughes WT. Treatment of pneumocystis carinii pneumonitis. $N$ Engl 7 Med $1976 ; 295: 726-7$

Reviews Pneumocystis carinii infection and treatment in the immunosuppressed patient.

Jackson D, Phillips I. From penicillin to piperacillin. $\mathcal{F}$ Antimicrob Chemother $1982 ; 9$, suppl B. 
A historical review of the development of penicillins with particular reference to the antimicrobial spectrum and clinical evaluation of piperacillin.

Keighley MRB, Burdon DW, Arabi Y, et al. Randomised controlled trial of vancomycin for pseudomembranous colitis and postoperative diarrhoea. Br Med f 1978; ii :1667-9.

This study showed that vancomycin was an effective and safe treatment for pseudomembranous colitis.

Ness HC. Current practices in antimicrobial dosing. Rev Infect Dis 1981 ;3: 12-8.

Reviews current practice relating to dosage and duration of antibiotic treatment.

Selwyn S. Mechanisms and range of activity of penicillin and cephalosporins. In: The 3 -lactam antibiotics: penicillins and cephalosporins in perspective. London: Hodder and Stoughton, 1980;1-55.
The antibacterial spectrum and mode of action of penicillins and cephalosporins are discussed.

Shaw EJ, Datta N, Jones G, Marr FM, Froud WJB. Effect of stay in hospital and oral chemotherapy on the antibiotic sensitivity of bowel coliforms. f Hygiene 1973;71:529-34.

Reviews the emergence of resistance in bowel coliforms during antibiotic treatment.

Swartz MN. Clinical aspects of legionnaires disease. Ann Intern Med 1979; 90:492-5.

Clinical features of legionnaires disease and its management are discussed.

Wise R, Reaves DS. Advances in therapy with antibacterial folate inhibitors. f Antimicrob Chemother $1979 ; 5$, suppl B.

Reviews the role and development of folate inhibitors.

\section{Clinical curio: perforating eye injury from an unusual cause}

Two young men in two months presented to the eye casualty department of the Leicester Royal Infirmary with perforating eye injuries from the same unusual cause. Both had been struck in the eye by a large fencing staple while they were pulling down nylon netting stapled to wooden posts. Both still have normal visual acuity.

Case 1-A 16 year old boy presented with a fencing staple $2 \mathrm{cms}$ long transfixing his left lower eyelid (fig $1 \mathrm{a}$ ). $X$ ray appearances (figs $1 b, 1 c)$ and clinical examination showed that the globe was perforated by one arm of the staple, the tip of which could be seen with a funduscope in the vitreous compartment. He had little discomfort and the visual acuity in the affected eye was $6 / 36$. The staple was removed

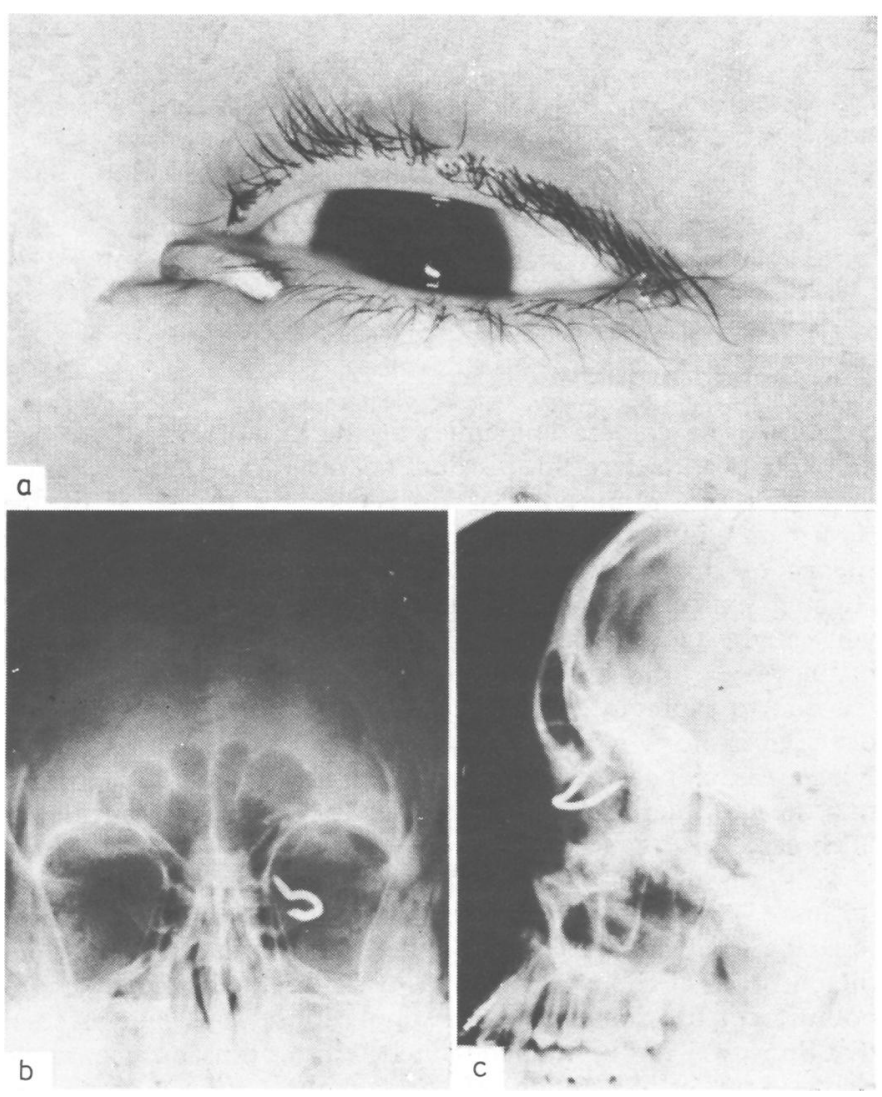

Clinical and $x$ ray appearances of staple injury to the left eye (case 1 ).

under general anaesthesia, requiring quite forceful traction to extract it. The perforation lay $6 \mathrm{~mm}$ posterior to the limbus. The scleral defect was sutured with 5/0 Dacron, and cryotherapy was applied around the wound and behind it. He was given intensive prophylactic local and systemic antibiotics. Seven days later he had regained left visual acuity of $6 / 9$ unaided. Fifteen months later the vision was $6 / 5$, the eye was not inflamed, and the retina was flat.
Case 2-A 21 year old man presented with a painful right eye after being struck by a fencing staple. Examination showed a full thickness puncture wound of the periphery of the right cornea, which was apparently self sealing; the anterior chamber was present but reduced. The iris was adherent to the back of the wound, though there was no prolapse of iris tissue through the wound. The lens was undamaged, and the visual acuity was $6 / 18$ in the affected eye. He was treated conservatively with topical and systemic antibiotics. The corneal wound healed well, and the anterior chamber reformed to normal depth. A small scar remains with iris anterior synaechias. Fourteen months later the visual acuity had returned to $6 / 5$.

No similar cases have been reported. $S m^{1}{ }^{1}$ and Blake ${ }^{2}$ described ocular injuries in agricultural workers, in whom perforating injuries by tools or metal are rare. Johnston ${ }^{3}$ reported a wider range of perforating injuries, but did not give all the causes, although, again, injuries with metal are rare in agricultural workers but prevalent in industrial workers. It is clear, especially in case 1 , that the staple was propelled with considerable force into the eye. The elastic nature of nylon netting presumably produced a catapult effect when the staple came free. Perforating puncture wounds of the eye, even when apparently severe, are compatible with the retention of good vision if promptly and appropriately treated. Perforating injuries where vegetable matter may contaminate the wound are vulnerable to infection, a devastating complication. Both these patients were treated vigorously with antibiotics prophylactically, and neither developed infection. Both were fortunate in the outcome of their injuries from this previously unrecognised hazard.

I should like to thank Miss D Callaghan for the photographic help, Mrs J Wood for her secretarial help, and Mr D B Goulstine and Mr D J Austin for permission to report these cases.-A B BECKINGSALE, ophthalmologist, Leicester.

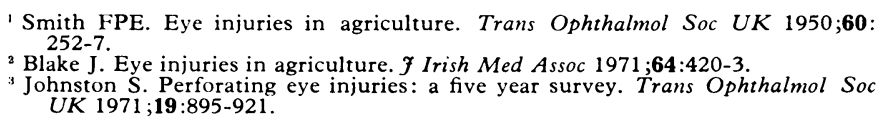

I find that circumcision of adults done under local anaesthesia using a basal ring block of xylocaine without adrenalin is satisfactory, except for some brief discomfort when cutting through the frenulum with scissors. Stitching this area causes few complaints. Can this discomfort be explained on anatomical and physiological grounds and can it be remedied?

The distribution of sensory nerve fibres from the dorsal nelve of the penis is particularly profuse not only to the glans penis but also to the skin of the frenulum. Indeed, this region is characterised by the presence within the papillae of the skin of specialised encapsulated sensory nerve terminals. The most likely explanation for the discomfort on cutting the frenulum relates to this rich innervation and suggests that the dorsal nerves of the penis are only partially blocked at the time of surgery. An indication of the efficiency of the block can be achieved by testing sensation over the surface of the glans. It seems unlikely that other nerves supplying the penis that lie deep to the site of injection could be responsible for persistent sensation of the frenulum. These autonomic cavernous nerves supply the vasculature of erectile tissue and are not considered to carry afferent nerves from the skin of the penis.-JOHN GOSLING, professor of anatomy, Manchester. 